\title{
Use of transoesophageal echocardiography to detect left atrial thrombi before percutaneous balloon dilatation of the mitral valve: a prospective study
}

\author{
Warren J Manning, Gregg J Reis, Pamela S Douglas
}

\begin{abstract}
Objective-Systemic emboli related to atrial thrombi are a well known complication of percutaneous balloon dilatation of the mitral valve. The presence of left atrial thrombi therefore, is believed to be a contraindication to balloon dilatation. The purpose of this study was to determine the frequency of left atrial thrombi in patients referred for balloon dilatation of the mitral valve, the added benefit of pre-procedural transoesophageal echocardiography, and to identify factors that predicted left atrial thrombi.
\end{abstract}

Design-Prospective study over a 14 month period of 20 consecutive patients by cross sectional transthoracic echocardiography $24-48$ hours before balloon dilatation of the mitral valve and by transoesophageal echocardiography immediately before the procedure.

Results-One patient had a left atrial thrombus detected by transthoracic study. Two patients $(10 \%)$ had left atrial thrombi identified by transoesophageal echocardiography. In both valve dilatation was not attempted and the thrombi were confirmed at surgery. The remaining 18 patients all underwent successful balloon dilatation of the mitral valve without clinical evidence of an embolic event. No association was found between patient age, mitral valve area, transmitral gradient, left atrial size, presence of atrial fibrillation, severity of mitral regurgitation, cardiac output, or the presence of left atrial swirling and an increased prevalence of atrial thrombi.

Conclusion-Left atrial thrombi are often seen despite long term systemic anticoagulation in patients referred for balloon dilatation of the mitral valve. The frequency of unsuspected left atrial thrombi detected by transoesophageal echocardiography was similar to the reported frequency of embolic events after balloon dilatation of the mitral valve. Transoesophageal echocardiography for the identification of left atrial thrombi is strongly recommended in all patients before balloon dilatation of the mitral valve including those treated with systemic anticoagulation and those who have had a normal transthoracic echocardiographic study.

The technique of percutaneous balloon dilatation of the mitral valve by the transseptal approach has been generally accepted as an alternative to mitral valve replacement or surgical commissurotomy in a subgroup of patients with symptomatic mitral stenosis. ${ }^{12}$ Mitral stenosis is frequently associated with left atrial thrombi ${ }^{3}$ and systemic emboli are a well known complication of balloon dilatation of the mitral valve (incidence $4 \%$ ). ${ }^{4}$ These events are believed to be caused primarily by embolisation of left atrial thrombi, when a thrombus is dislodged during the procedure. So the presence of left atrial thrombi is thought to be a contraindication to balloon dilatation of the mitral valve. Recently, transoesophageal echocardiography has been shown to be much better than conventional cross sectional transthoracic echocardiography for the identification of left atrial thrombi. ${ }^{5-7}$ The purpose of this study was to evaluate the role of routine transoesophageal echocardiography for the identification of left atrial thrombi before the procedure.

\section{Patients and methods}

PATIENTS

We studied 20 consecutive patients ( 18 women and two men aged $41-79$ years (mean 59 years)) referred for elective balloon dilatation of the mitral valve from 1 December 1989 to 28 February 1991. Three patients had undergone earlier surgical commissurotomies and nine patients were in chronic atrial fibrillation. All 20 patients had been treated with sodium warfarin (therapeutic prothrombin times $>1.5$ times control) for at least eight weeks before planned balloon dilatation of the mitral valve. No patient had a history of an earlier embolic event. Coumadin was stopped two days before admission. The identification of an atrial thrombus was thought to be an indication for mitral valve replacement. Patients undergoing balloon dilatation of the mitral valve were followed clinically throughout their stay in hospital for evidence of systemic emboli. 
Written informed consent was obtained from all patients.

\section{TRANSTHORACIC ECHOCARDIOGRAPHY}

Patients underwent conventional transthoracic cross sectional and Doppler echocardiographic examination 12-36 hours before the planned balloon dilatation of the mitral valve by techniques reported previously. ${ }^{8}$ Cross sectional imaging studies were performed in the parasternal long axis, parasternal short axis, apical, and subcostal views by a Sonos 500 or Sonos 1000 (Hewlett-Packard) echocardiograph and both $2.5 \mathrm{MHz}$ and $5.0 \mathrm{MHz}$ phased array transducers. The left atrial anteroposterior diameter was measured in the parasternal long axis view according to the American Society of Echocardiography criteria. ${ }^{9}$ Left atrial length was assessed in the four chamber apical view. Mitral valve area was assessed by the pressure half time technique. ${ }^{8}$ Spontaneous contrast was defined as the presence of dynamic echoes (smoke-like) in a circular pattern within the left atrium. ${ }^{10}$

\section{TRANSOESOPHAGEAL ECHOCARDIOGRAPHY}

All patients underwent transoesophageal echocardiography examination after an overnight fast and within two hours of the scheduled balloon dilatation of the mitral valve $(n=18)$ or the day before the planned procedure $(n=2)$. Local anaesthesia to the posterior pharynx was accomplished with $10 \%$ lignocaine spray. Antibiotics for endocarditis prophylaxis were given to all patients according to the recommendations of the American Heart Association. " Transoesophageal echocardiography was performed with a commercial single plane $5.0 \mathrm{MHz}$ transoesophageal echocardiography probe (Hewlett-Packard). Images of the cardiac chambers, valves, and great vessels were recorded on videotape for review. Special attention was paid to the left atrium and the left atrial appendage.

BALLOON DILATATION OF THE MITRAL VALVE Patients in whom there was no evidence of atrial thrombi by transthoracic or transoesophageal echocardiography underwent balloon dilatation of the mitral valve with a single $25 \mathrm{~mm}$ Mansfield balloon (Mansfield Scientific, Waltham, Massachusetts) by the transseptal approach. ${ }^{2}$ Fick cardiac output was obtained in all patients before placement of the transseptal needle. ${ }^{12}$

Six additional patients with rheumatic mitral stenosis underwent transoesophageal echocardiographic study to identify left atrial thrombi while under general anaesthesia in the operating room before mitral valve replacement. These patients were not candidates for balloon dilatation of the mitral valve because there was concomitant aortic stenosis $(n=2)$ or important mitral regurgitation $(n=4)$.

\section{DATA ANALYSIS}

All data are expressed as mean (SD). The statistical significance of left atrial size, Doppler variables, and catheterisation cardiac output/ index was assessed by Student's unpaired $t$ test.
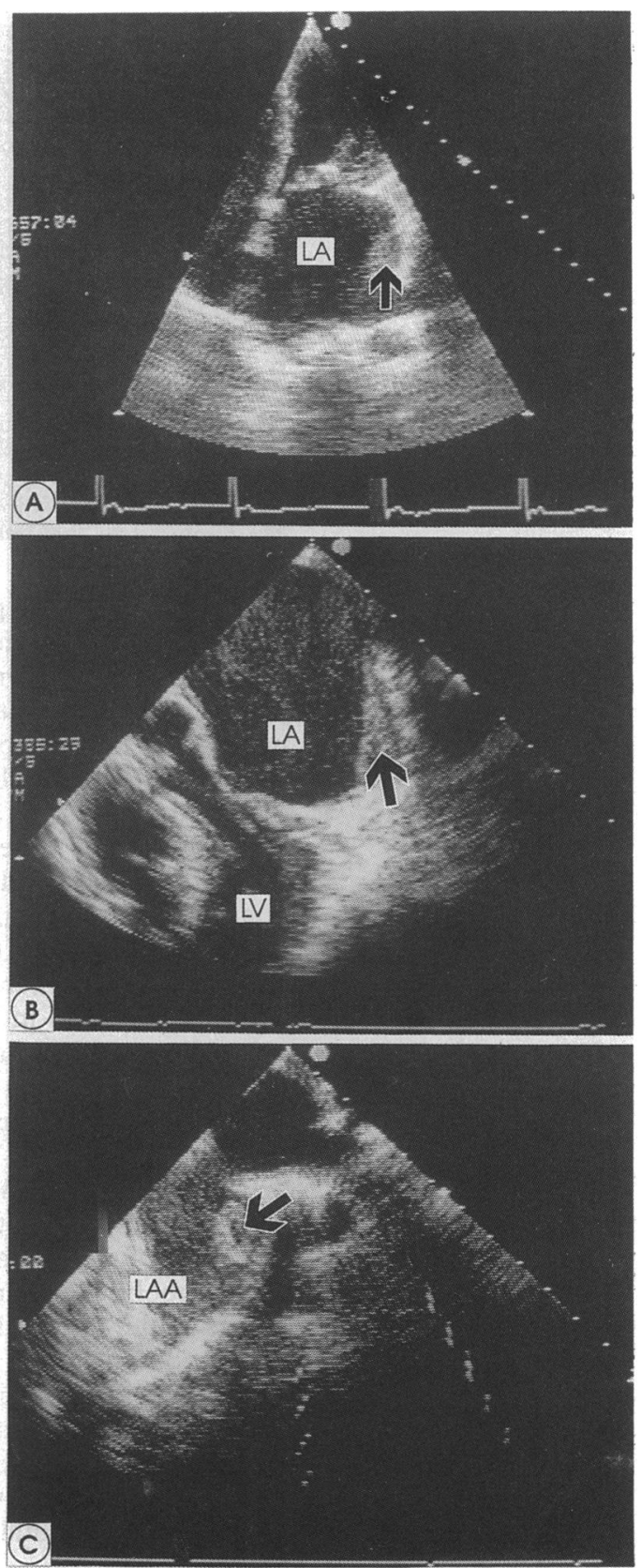

Figure 1 (A) Transthoracic apical four-chamber view of the heart obtained with a $5 \mathrm{MHz}$ imaging transducer. Note the thrombus (arrow) along the lateral wall of the left atrium (LA). (B) Transoesophageal

echocardiography. Note the thrombus (arrow) lining the lateral wall of the left atrium and spontaneous contrast within the left atrium. $L V$, left ventricle.

(C) Transoesophageal echocardiography study of the left atrial appendage (LAA) of the same patient shown in $A$ and $B$. Note the second thrombus (arrow).

Fischer's exact test was used to compare dichotomous variables. A p value of $<0.05$ was considered statistically significant.

\section{Results}

Transthoracic echocardiographic study identified a probable thrombus in one patient scheduled for balloon dilatation. The thrombus was located along the lateral wall of the left atrium (fig 1A).

All patients tolerated transoesophageal echocardiography without complications. Imaging of the left atrial appendage was 
Figure 2

Transoesophageal echocardiography view of the left atrium in another patient. Note the thrombus (arrow) in the distal portion of the left atrial appendage.

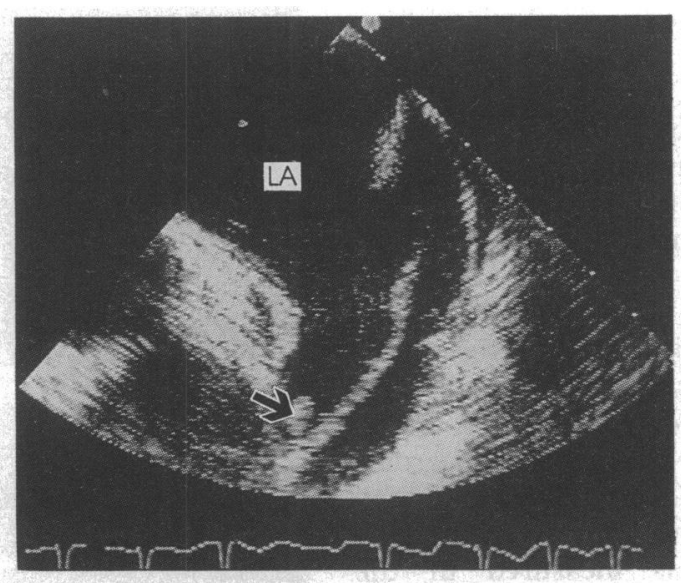

successful in all 18 patients in whom it was present. In two patients the left atrium had been either oversewn $(n=1)$ or amputated ( $n=1)$ during an earlier surgical commisurotomy. Transoesophageal echocardiography confirmed the thrombus seen by transthoracic study (fig 1B) and also identified a second thrombus in this patient, which was confined to the left atrial appendage (fig 1C). Transoesophageal echocardiography also identified a thrombus confined to the distal left atrial appendage in another patient (fig 2). We found left atrial thrombi in $10 \%$ of patients referred for balloon dilatation of the mitral valve. In both instances in which a thrombus was identified patients were referred for mitral valve replacement and the site and presence of all thrombi were confirmed at the time of surgery. The remaining 18 patients underwent balloon dilatation of the mitral valve without evidence of an embolic event during their hospital stay.

The table shows the characteristics of the subgroups with and without atrial thrombi. Patient age, atrial fibrillation, mitral valve area, mitral valve gradient, cardiac output/index, severity of mitral regurgitation, and presence of left atrial swirling were not predictive of thrombi (table). Total echo score ${ }^{13}$ was also not predictive.

No thrombi were identified by transoesophageal echocardiographic study in any of the patients with rheumatic mitral stenosis scheduled for mitral valve replacement. The table shows the characteristics of this group of patients. Visual inspection of the left atrium also failed to reveal any thrombi.

Comparison of echocardiographic and catheterisation data in patients with left atrial thrombi and without

\begin{tabular}{|c|c|c|c|}
\hline Variable & $\begin{array}{l}\text { With LA thrombus } \\
(n=2)\end{array}$ & $\begin{array}{l}\text { No LA thrombus } \\
(\text { dilatation }) \\
(n=18)\end{array}$ & $\begin{array}{l}\text { No LA thrombus } \\
\text { (replacement }) \\
(n=6)\end{array}$ \\
\hline $\begin{array}{l}\text { Age (yr) } \\
\text { Atrial fibrillation } \\
\text { Left atrial dimension }(\mathrm{cm}) \\
\text { Four chamber left atrial length }(\mathrm{cm}) \\
\text { Mitral valve area }\left(\mathrm{cm}^{2}\right) \\
\text { Mitral valve gradient }(\mathrm{mm} \mathrm{Hg}) \\
\text { Cardiac output }(1 / \mathrm{min}) \\
\text { Cardiac index }\left(1 / \mathrm{min} / \mathrm{m}^{2}\right) \\
\text { Severity of } \mathrm{MR}(0-3) \\
\text { Left atrial swirling (TTE) } \\
\text { Left atrial swirling (TOE) } \\
\text { Echo score }(0-16)\end{array}$ & $\begin{array}{l}52(41-63) \\
2(100 \%) \\
5 \cdot 4(0 \cdot 5) \\
6 \cdot 5(0 \cdot 2) \\
0 \cdot 7(0 \cdot 1) \\
8 \cdot 8(0 \cdot 3) \\
4 \cdot 3(0 \cdot 2) \\
2 \cdot 7(0 \cdot 2) \\
1 \cdot 3(0 \cdot 4) \\
1(50 \%) \\
1(50 \%) \\
8(3)\end{array}$ & $\begin{array}{l}60(41-79) \\
7(39 \%) \\
5 \cdot 4(0 \cdot 7) \\
6 \cdot 4(1 \cdot 0) \\
1 \cdot 1(0 \cdot 4) \\
8 \cdot 5(3 \cdot 4) \\
4 \cdot 5(1 \cdot 4) \\
2 \cdot 7(0 \cdot 7) \\
0 \cdot 9(0 \cdot 5) \\
3(15 \%) \\
6(33 \%) \\
6(2)\end{array}$ & $\begin{array}{c}59(40-67) \\
4(67 \%) \\
5.9(0 \cdot 2) \\
7.7(1 \cdot 1) \\
1 \cdot 1(0 \cdot 6) \\
9.0(5 \cdot 0)\end{array}$ \\
\hline
\end{tabular}

${ }^{\star} \mathrm{p}<0.05 v$ no LA thrombus (dilatation). MR, mitral regurgitation; TOE, transoesophageal echocardiogram; TTE, transthoracic echocardiogram.

\section{Discussion}

Systemic embolisation is a recognised complication of balloon dilatation of the mitral valve. It occurs in up to $4 \%$ of patients. ${ }^{4}$ The ability to identify and exclude those patients at high risk of systemic emboli would significantly reduce the morbidity of this procedure. Transthoracic echocardiography has a low sensitivity for detection of left atrial thrombi, particularly those in the left atrial appendage. ${ }^{7}$ Transoesophageal echocardiography gives superior visualisation of the appendage, and previous studies showed it was a more sensitive detector of left atrial thrombi. ${ }^{56}$

The present study shows that transoesophageal echocardiography can be safely performed and can accurately detect and localise left atrial thrombi in patients with severe mitral stenosis referred for balloon dilatation of the mitral valve. Despite chronic systemic anticoagulation $10 \%$ of patients studied had left atrial thrombi which we confirmed at surgery. Our findings are consistent with those of Kronzon et al who reported that $26 \%$ of patients undergoing transoesophageal echocardiography before balloon dilatation of the mitral valve had a detectable left atrial clot. ${ }^{14}$ The lower frequency of thrombi in our study may be related to the use of at least two months of systemic anticoagulation in our patients. Kronzon et al, however, did not examine risk factors that were potentially predictive for the development of atrial thrombi. Low cardiac output and age have been reported as risk factors for systemic emboli in patients with mitral stenosis. ${ }^{3}$ Our findings suggest that patient age, atrial fibrillation, left atrial size, mitral valve gradient, cardiac output, severity of mitral regurgitation, left atrial swirling, and echo score did not predict the presence of atrial thrombi in our group of anticoagulated patients. There was a trend towards a smaller mitral valve area in the group with atrial thrombi but this did not achieve statistical significance. Thus we were unable to identify any descriptors that might be useful in deciding which patients, if any, are at sufficiently low risk for left atrial thrombi that a transoesophageal echocardiography may be omitted.

Spontaneous left atrial contrast in mitral stenosis has been suggested as an indicator for an increased risk of thromboembolism ${ }^{15}$ and atrial thrombosis. Spontaneous contrast is felt to be related to the rouleaux formation of erythrocytes which occur during slow flow conditions. ${ }^{16}$ In vitro and in vivo experiments showed that blood within isolated vessels may become echogenic within a few seconds after the onset of stasis induced by cross clamping. ${ }^{16}$ In addition, fibrinogen or its products plus red blood cells are needed to create echogenicity of static liquid blood. This phenomenon is not prevented by heparin. We did not find the presence of spontaneous left atrial contrast to be predictive for left atrial thrombi. Our study population, however, was small and all our patients were systemically anticoagulated.

Patients with left atrial thrombi identified by transthoracic study are usually not referred for 
balloon dilatation of the mitral valve. The additional $5 \%$ frequency of left atrial thrombi identified by transoesophageal echocardiography in our study is similar to the reported incidence of embolic events with balloon dilatation reported in large series. ${ }^{4}$ Most patients in these large series have not undergone pre-procedural transoesophageal echocardiography. While the exact frequency of embolisation in patients with atrial thrombi is unknown, it is reasonable to assume that they are at higher risk than patients without thrombi.

\section{LIMITATIONS}

Although systemic emboli are often related to dislodgement of atrial thrombi, some of these events may be due to thrombi forming on catheters and guide wires, to debris released with commissure fracture, ${ }^{2}$ or to balloon rupture. ${ }^{17}$ These events would not be avoided by a pre-procedural transoesophageal echocardiography. A decreased incidence of embolic events also cannot be estimated by this study. While no subject in our study had evidence of systemic emboli, a much larger study will be needed to determine whether patients with negative transoesophageal echocardiography examinations have a lower risk of embolisation or if the frequency of embolic events would be reduced if balloon dilatation of the mitral valve were avoided in patients with thrombi identified by transoesophageal echocardiography.

\section{CONCLUSION}

Left atrial thrombi are often seen despite long term systemic anticoagulation in patients referred for balloon dilatation of the mitral valve. The frequency of unsuspected left atrial thrombi detected by transoesophageal echocardiography is similar to the incidence of embolic events reported with balloon dilatation.

We strongly recommend transoesophageal echocardiography to identify left atrial thrombi before balloon dilatation of the mitral valve, including patients treated with systemic anticoagulation and those who have had a normal transthoracic study. The identification of a left atrial thrombus should be considered a contraindication to the balloon dilatation of the mitral valve and such patients should be referred for mitral valve replacement or open surgical commissurotomy.

W J M is supported in part by a Physician-Scientist Award from the National Institute of Aging, Baltimore, Maryland, USA. We are grateful for the technical assistance of Marilyn F Riley, RDCS.

1 Inoue $\mathrm{K}$, Owaki T, Nakamara T, Kitamura F, Miamoto N. Clinical application of transvenous mitral commissurotomy by a new balloon catheter. J Thorac Cardiovasc Surg 1984;87:394-402.

2 McKay RG, Lock JE, Safian RD, et al. Balloon dilation of mitral stenosis in adult patients: postmortem and percutaneous mitral valvuloplasty studies. J Am Coll Cardiol 1987;9:723-31.

3 Braunwald E. Valvular heart disease. In: Braunwald E, ed. Heart disease. 3rd ed. Philadelphia: WB Saunders, 1988:1023-34.

4 Kulick DL, Reid CL, Kawanishi DT, Rahimtoola SH. Catheter balloon commissurotomy in adults. Part II: Mitral and other stenoses. Curr Probl Cardiol 1990; 15:397-470.

5 Aschenberg W, Schlüter $M$, Kremer P, Schröder E, Siglow V, Bleifeld $W$. Transesophageal two-dimensional echocardiography for the detection of left atrial appendage thrombus. J Am Coll Cardiol 1986;7:163-6.

6 Pearson AC, Labovitz AJ, Tatineni S, Gomez CR. Superiority of transesophageal echocardiography in detecting cardiac source of embolism in patients with cerebral ischemia of uncertain etiology. J Am Coll Cardiol 1991;17:66-72.

7 Shrestha NK, Moreno FL, Narciso FV, Torres L, Calleja HB. Two-dimensional echocardiographic diagnosis of left atrial thrombus in rheumatic heart disease: a clinicopathologic study. Circulation 1983;67:341-7.

8 Come PC, Riley MF, Diver DJ, Morgan JP, Safian RD McKay RG. Noninvasive assessment of mitral stenosis before and after percutaneous balloon mitral valvulobefore and after percutaneous balloon
plasty. Am J Cardiol 1988;61:817-25.

9 Sahn DJ, DeMaria A, Kisslo J, Weyman A. Recommendations regarding quantitation in $\mathrm{M}$-mode echocardiography: results of a survey of echocardiographic

10 Castello R, Pearson AC, Labovitz AJ. Prevalence and clinical implications of atrial spontaneous contrast in patients undergoing transoesophageal echocardiography. Am J Cardiol 1990;65:1149-53.

1 Shulman ST, Amren DP, Bisno AL, et al. Prevention of bacterial endocarditis. Circulation 1984;70:1123A-7A.

12 Grossman W. Blood flow measurement: the cardiac output In: Grossman W, ed. Cardiac catheterization and angiography. 3rd ed. Philadelphia: Lea \& Febinger, 1986: $101-17$.

13 Wilkins GT, Weyman AE, Abascal VM, Block PC, Palacios IF. Percutaneous balloon dilatation of the mitral valve: an analysis of echocardiographic variables related to outcome and the mechanism of dilatation. Br Heart J 1988;60: 299-308.

14 Kronzon I, Tunick PA, Glassman E, Slater J, Schwinger M Freedberg RS. Transesophageal echocardiography to detect atrial clots in candidates for percutaneous transdetect atrial clots in candidates for percutaneous transseptal mitral ball

15 Daniel WG, Nellessen U, Schröder E, et al. Left atrial spontaneous echo contrast in mitral valve disease: an indicator for an increased thromboembolic risk. J Am Coll Cardiol 1988;11:1204-11.

16 Sigel B, Coelho JCU, Spigos DG, et al. Ultrasonography of blood during stasis. Invest Radiol 1981;16:71-6.

17 Vahanian A, Michel PL, Cormier B, et al. Results of percutaneous mitral commissurotomy in 200 patients. $A m$ J Cardiol 1989;63:847-52. 